\title{
Applications of Environmental Scanning Electron Microscopy (ESEM) in Pharmaceutical Development
}

\author{
J.P. Neilly, ${ }^{*}$ A.D. Vogt, ${ }^{*}$ L.A. Sciaraffa, ${ }^{*}$ S.Z. Ariman, ${ }^{* *}$ D.R. Willcox, ${ }^{* *}$ J.A. Fagerland* \\ * Abbott Laboratories, Exploratory and Investigative Technology (R45M, AP31), \\ Abbott Park, IL 60064-6202 \\ ** Abbott Laboratories, Process Development (R452, R13), North Chicago, IL 60064
}

The introduction of the environmental scanning electron microscope (ESEM) permitted the examination of specimens in a gaseous environment [1]. ESEM has been used to reduce charging problems associated with pharmaceutical powders [2], and to study hydration of pharmaceutical excipients [3]. The ESEM has the advantage of extremely small sample requirements. Using the ESEM, we have been able to study changes in crystal morphology of active pharmaceutical ingredients (APIs) during hydration and dehydration in the specimen chamber.

Water was used as the ionizing gas in these experiments, and changes in crystal morphology were correlated with relative humidity $(\mathrm{RH})$. Samples were sprinkled onto the standard Peltier stage of an FEI XL30 ESEM. The stage was cooled to $5-10^{\circ} \mathrm{C}$ and allowed to stabilize under high vacuum for 10-30 minutes. During hydration, the RH was raised from $30 \% \mathrm{RH}$ to $90 \% \mathrm{RH}$ by increasing the chamber pressure in 1.0 torr increments. Chamber pressure was further increased in 0.1 torr increments to $100 \% \mathrm{RH}$ or until water condensed on the particles. After condensation, the particles were dehydrated by decreasing the chamber pressure in 1.0 torr increments. Particles were examined and photographed during hydration and dehydration steps.

An example of two lots of the same API are shown in Figures 1 and 2. In the first lot, small droplets formed on the particle surface during hydration. The droplets disappeared during dehydration leaving large surface cracks on the crystal (Figure 1). In a second lot of the same compound, droplets also formed on crystal surfaces during hydration (Figure 2). However, very little surface damage was present after dehydration. Karl Fisher analysis of the first lot showed that it had higher water content. Thus, the ESEM was able to detect morphology differences in two lots of the same API that correlated with water content in the bulk material.

This example shows that the ESEM is a valuable tool for studying APIs during pharmaceutical development. The instrument can easily image hygroscopic powders without the use of conductive coatings, but, more importantly, the ESEM can be used to study the microscopic behavior of APIs in response to wet conditions and, potentially, to identify unstable crystal compounds. The ESEM can detect microscopic properties of hydrated APIs that can correlate to macroscopic behavior.

[1] G.D. Danilatos, Microscopy Research and Technique 25 (1993) 354.

[2] M.J. Clarke, et al., Pharmacy and Pharmacology Communications 4 (1998) 419.

[3] R.A. Roberts, A.J. Shukla, and T. Rice, Scanning 19 (1997) 104. 

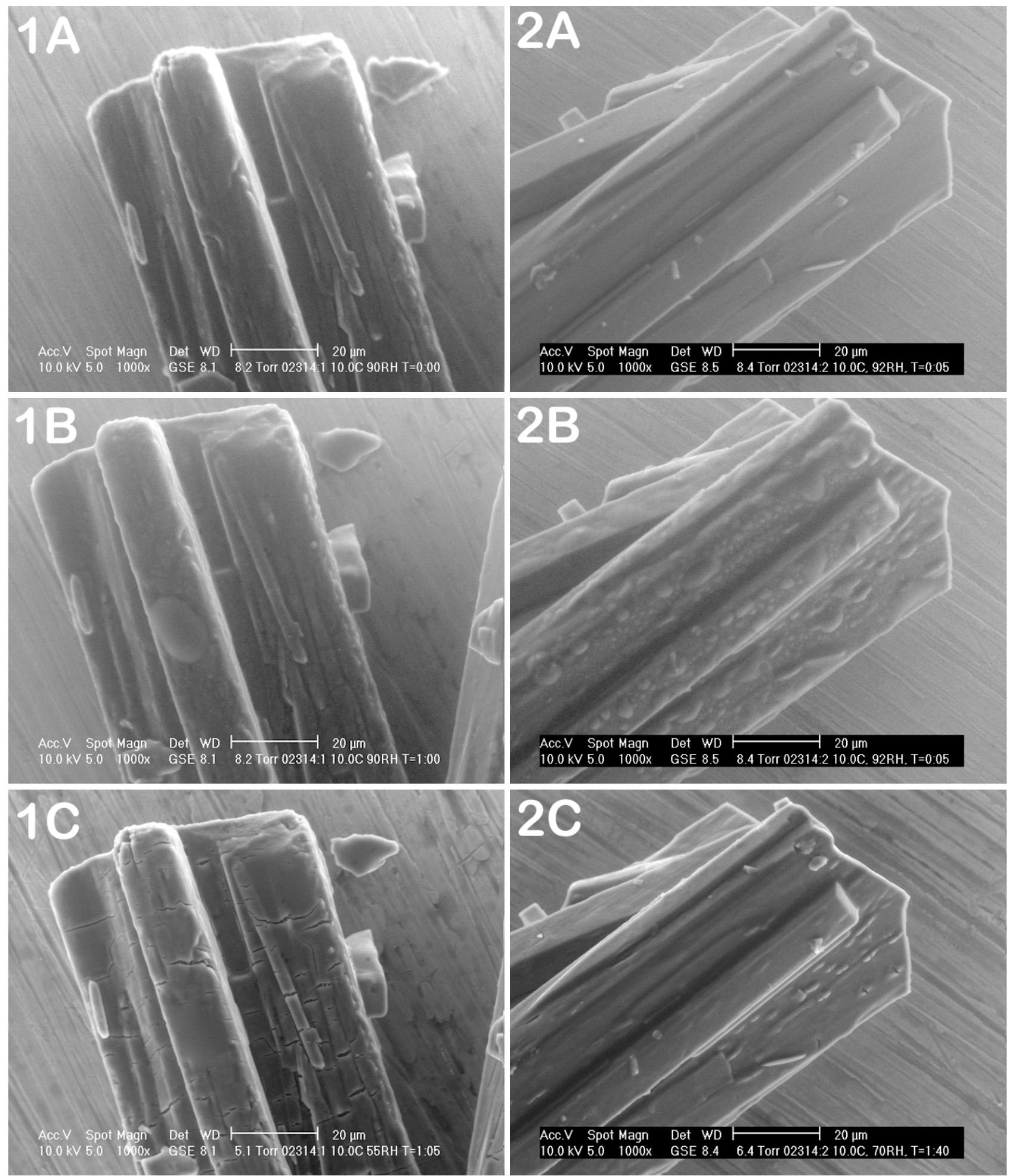

Figure 1. Particle morphology of unstable lot before hydration (1A), during hydration (1B), and after dehydration (1C).

Figure 2. Particle morphology of stable lot before hydration (2A), during hydration (2B), and after dehydration $(2 \mathrm{C})$. 